\title{
Prosthesis preference is related to stride-to-stride fluctuations at the prosthetic ankle
}

\author{
Shane R. Wurdeman, CP, MSPO; ${ }^{1-2}$ Sara A. Myers, PhD; ${ }^{1}$ Adam L. Jacobsen, CPO; ${ }^{3}$ Nicholas Stergiou, PhD $^{1-2 *}$ \\ ${ }^{1}$ Nebraska Biomechanics Core Facility, University of Nebraska at Omaha, Omaha, NE; ${ }^{2}$ College of Public Health, \\ University of Nebraska Medical Center, Omaha, NE; ${ }^{3}$ Department of Prosthetics and Sensory Aids, Department of Vet- \\ erans Affairs Nebraska-Western Iowa Health Care System, Omaha, NE
}

\begin{abstract}
The purpose of this study was to determine the relationship between stride-to-stride fluctuations and prosthesis preference. Thirteen individuals with unilateral, transtibial amputation consented to participate. Individuals walked on a treadmill for 3 min with their prescribed and an alternate prosthesis. Stride-to-stride fluctuations were quantified with the largest Lyapunov exponent (LyE) of each joint flexion/extension time series. The change in the LyE was calculated for each major lower-limb joint for both conditions. Participants indicated preference between the prostheses on a continuous visual analog scale. The change in the LyE was correlated with the degree of preference between the two prostheses at the prosthetic ankle. The change in the LyE of the prosthetic ankle was strongly related to the degree of preference $(r=0.629, p=$ 0.02 ). Thus, stride-to-stride fluctuations, quantified by the LyE, are strongly related to the patient's perception of the prosthesis. As a result, the LyE is the first objective measure to detect changes in gait that relate to the patient's perception of the prosthesis. The LyE should be further examined as a potentially effective prescriptive and outcome measure in prosthetic rehabilitation.
\end{abstract}

Key words: amputation, dynamic stability, dynamical system, gait, limb loss, Lyapunov exponent, nonlinear dynamics, outcomes, perception, rehabilitation, transtibial, variability.

\section{INTRODUCTION}

There are approximately 1.6 million Americans living with limb loss, a number projected to reach 3.6 million by the year 2050 [1]. From 1988 to 1996, there were on average nearly 134,000 hospital discharges for amputations per year; 82 percent of these discharges were lower-limb amputations [2]. The growth of this population increases pressure to provide quality prosthetic rehabilitation enhanced by the most appropriate prosthesis. Unfortunately, the most effective tool for lower-limb prosthesis prescription that is available for the rehabilitation team is the Amputee Mobility Predictor [3]. The Amputee Mobility Predictor is only designed to determine a patient's current and potential functional level based on Medicare classifications; it is not able to provide guidelines on the specific prescription of a prosthesis. In regards to specific prosthetic prescription, clinicians must largely rely upon history, physical examination, and experience of the rehabilitation team, which may incorporate any available empirical evidence [4-5]. Outcomes for prosthetic rehabilitation must rely upon the clinician's report and even more on satisfactory patient perception [6]. This may be problematic as the information provided by the patient may be

\footnotetext{
Abbreviations: $\mathrm{Ax}=$ alternate, $\mathrm{ESAR}=$ energy-storage-andreturn (prosthetic foot), LyE = largest Lyapunov exponent, $\mathrm{MTP}=$ metatarsophalangeal, $\mathrm{Rx}=$ prescribed.

*Address all correspondence to Nicholas Stergiou, PhD; Nebraska Biomechanics Core Facility, University of Nebraska at Omaha, Omaha, NE 68182-0216; 402-5543247; fax: 402-554-3693. Email: nstergiou@unomaha.edu http://dx.doi.org/10.1682/JRRD.2012.06.0104
} 
cases. Ultimately, some individuals are simply better able to provide useful information.

Failure to achieve a strong prescriptive tool may in part be due to the lack of an objective measure relating to patient perception. Patients consistently prefer energystorage-and-return (ESAR) prosthetic feet [6-8], but the typical biomechanical metrics used to assess gait do not always demonstrate superiority of ESAR. Furthermore, Kark and Simmons showed a lack of relationship between gait deviations and prosthesis satisfaction, implying the role of gait is not unambiguously coupled to prosthesis satisfaction [9]. Numerous biomechanics measures covering all areas of kinematics and kinetics as well as electromyography and energy expenditure have been explored in gait of individuals with lower-limb amputation [6]. Yet, in a recent review, Hafner et al. stated, "... while the collected perceptive data appears to validate the strong clinical support afforded to ESAR prostheses, biomechanical results are often insufficient to name one type of prosthesis superior to another." [6].

It is possible that the changes in gait that affect the patient's perception do not lie within a single stride, but rather in the stride-to-stride fluctuations that occur over multiple strides. Specifically, standard measures in biomechanics will take an event within the gait cycle and average multiple steps to acquire a representative performance. While this technique is informative, it fails to account for the inherent relationship between strides that calculations such as the largest Lyapunov exponent (LyE) will capture. Measures, such as those utilized by Kark and Simmons [9], presume that every step is completely independent of the previous steps and has no influence on any ensuing steps. However, studies have confirmed the presence of strong relationships between continuous strides during gait [10-12]. This concept is somewhat intuitive when considering the example of a misstep, where it is routinely observed that an individual may need several steps before the gait pattern appears to be "normal" again [13]. But the misstep that caused such fluctuation from one specific stride to the next is actually an extreme example. In fact, there are natural fluctuations that occur within every stride-to-stride cycle that are considered to be a characteristic of nondisabled gait [12]. These fluctuations are the result of the neuromuscular system's optimization of all the different variables affecting the movement $[11,14-15]$. As a result, it is possible that previous measures that failed to relate to prosthesis preference may not have been the best reflection of neu- romuscular function. Prosthesis preference is a measure that falls within the realm of psychophysics: quantifying the perception of the individual with limb loss toward a physical stimulus [16]. Thus, the perception of the individual with limb loss may relate to the changes in strideto-stride fluctuations in the gait cycle and these may better quantify the aspect of neuromuscular control related to prosthesis preference [11].

In order to assess the fluctuations from stride-tostride, one must measure multiple consecutive strides and then analyze the entire time series rather than discrete points. A time series is a sequence of measurements ordered with respect to time. Figure 1 displays a time series for an ankle dorsiflexing and plantarflexing. Time is plotted on the abscissa such that at any given time during the walking trial, it is possible to determine the ankle angle. Viewing such a time series also makes it possible to see the oscillatory nature of gait. Furthermore, it is possible to see that the steps are similar, but no two steps are ever identical. Through time series analysis, it is possible to gain insight into the nature of these stride-tostride fluctuations.

Consequently, if these stride-to-stride fluctuations that naturally occur are influencing the patient's percep-

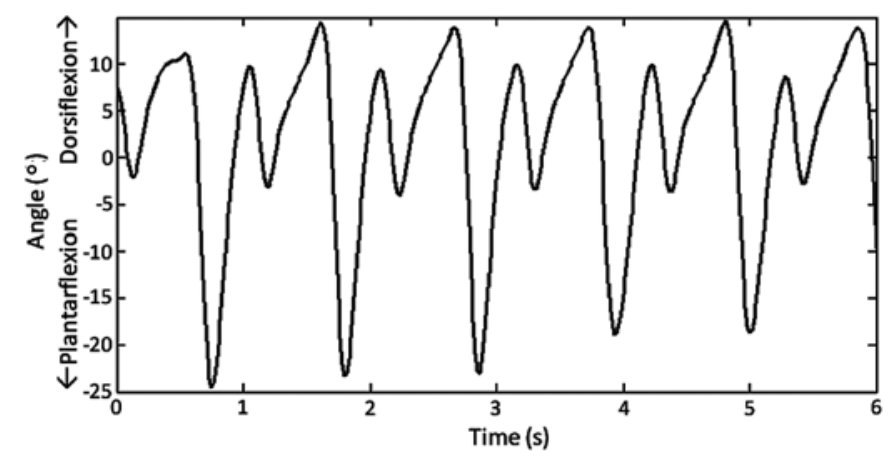

Figure 1.

Time series for one subject's ankle angle while walking. Plot displays angle continuously from 0 to $6 \mathrm{~s}$ of nonstop walking. Slightly $>5$ strides shown. Traditional measures would consider performance of individual as average of these 5 strides. However, such an approach would not account for temporal relationship held within order of these strides. Time series analysis utilizing nonlinear dynamical tools, such as largest Lyapunov exponent, is able to quantify this relationship. In case of limb-loss rehabilitation, such temporal relationship is affected by prosthesis. 
tion, then switching to a different prosthesis to evoke a change in such fluctuations should affect the patient's perception of the prostheses. In essence, a greater change in these fluctuations will cause increased deviation from the individual's inherent movement pattern. This would be expected to be accompanied by stronger feelings of preference between the prostheses. Therefore, the purpose of this study was to quantify the stride-to-stride fluctuations through the LyE during gait for patients with a lower-limb amputation walking in two separate prosthesis setups. The LyE has been used in the gait literature to quantify changes in these fluctuations [17-21]. The LyE was chosen (as opposed to other metrics such as detrended fluctuation analysis or sample entropy) specifically for its distinct benefits of measuring divergence of cycles within the attractor, making it ideal for investigating variability in joint kinematic patterns, which are inherently periodic [21-22]. This inherent periodicity can largely influence other metrics and dominate their outcomes when we are truly interested in the fluctuations that exist on top of these intercycle periodic dynamics. The difference in the LyE between the two prosthesis setups was then correlated with the patient's preference as denoted on a continuous visual analog scale. It was hypothesized that the change in the LyE would correlate to the degree of preference between prostheses.

\section{METHODS}

\section{Participants}

Fourteen individuals with lower-limb amputation were recruited for this study. Thirteen (eleven males, two females) were able to complete all tasks and thus were included for analysis (Table 1). Inclusion criteria included (1) unilateral transtibial level amputation, (2) ability to ambulate nonstop without any assistive device (other than the prosthesis) for $3 \mathrm{~min}$, (3) amputation for at least $1 \mathrm{yr}$, and (4) possession of current prosthesis longer than $30 \mathrm{~d}$. Exclusion criteria included presence of (1) any ulcers on either the residual limb or contralateral limb and (2) any neuromuscular or musculoskeletal conditions (beyond amputation and diabetes) that may affect gait.

\section{Gait Analysis Procedures}

Participants walked on a treadmill for $3 \mathrm{~min}$ at the same speed (self-selected preferred) for both conditions. Preferred walking speed was chosen because walking at

Table 1.

Characteristics of individuals able to complete tasks. All individuals used energy-storage-and-return feet during tasks.

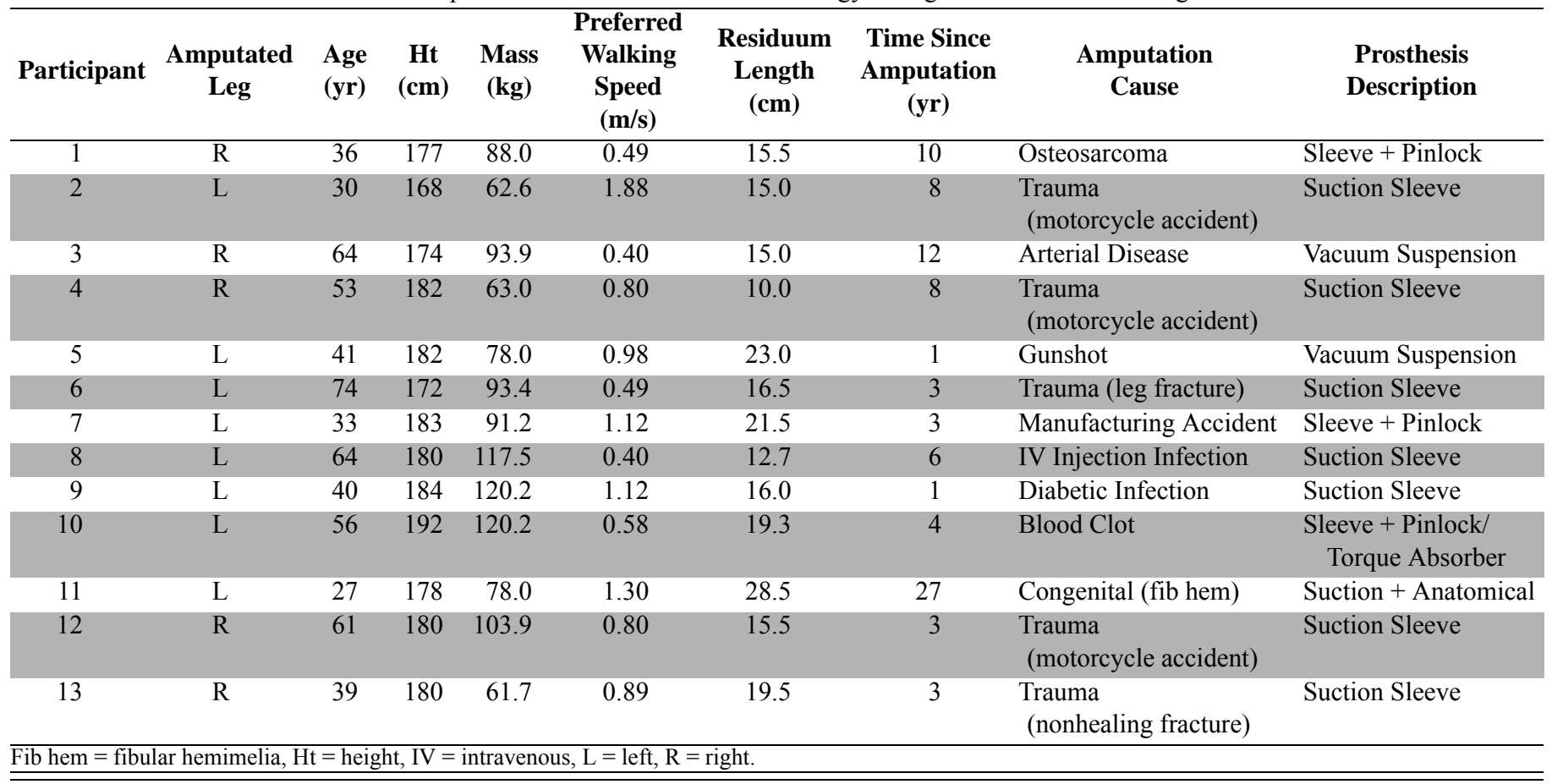


other speeds has been shown to affect an individual's stride-to-stride fluctuations in gait [23]. Preferred walking speed was determined by having the participant walk on the treadmill in his or her prescribed ( $\mathrm{Rx}$ ) prosthesis while the speed was incrementally increased until preferred walking speed was reached. Once the participant reported the speed had reached a comfortable, preferred walking speed, the subject ambulated another minute to confirm the proper speed had been selected. Following selection of preferred walking speed, subjects were required to rest for at least $1 \mathrm{~min}$. Participants performed two separate trials presented in random order, one with their Rx prosthesis and another with an alternate $(\mathrm{Ax})$ prosthesis. Between trials, subjects were again given adequate rest time, at least $1 \mathrm{~min}$, to minimize confounding effects of fatigue. The Rx prosthesis for all subjects included ESAR model feet. For the Ax prosthesis, all components distal to the socket were removed and replaced with a standard aluminum pylon and a solid-ankle-cushion-heel type foot (The Ohio Willow Wood Company; Mt. Sterling, Ohio). None of the participants had cosmetic covers, which would have possibly limited access to distal components. The pylons for the Rx prostheses were either aluminum, titanium, or carbon fiber. The Ax prosthesis was then properly aligned by a certified prosthetist consistent with common practice [24]. This method preserved the socket fit and suspension for each individual. Utilization of the individual's custom socket and suspension also allowed for quick alignment of the Ax prosthesis ( $<20 \mathrm{~min})$. Subjects were not allowed to walk for an extended period in order to maximize the perturbation to the stride-to-stride fluctuations present in the gait cycle. At the time of testing, all subjects had adequate fit and suspension consistent with common practice verified clinically by a certified prosthetist [24].

Subjects wore a tight-fitting uniform during data collection. Twenty-seven retroreflective markers were placed on anatomical locations on bilateral lower limbs and the pelvis such that each segment had a minimum of three markers permitting joint angle calculations as outlined by Vaughan et al. [25]. Markers were located bilaterally at (1) anterior superior iliac spines, (2) posterior superior iliac spines, (3) greater trochanters, (4) midlateral thighs, (5) lower front thighs, (6) lateral knees, (7) tibial tubercles, (8) lower lateral shanks, (9) lateral ankles, (10) top of second metatarsophalangeal (MTP) joints, (11) posterior heels, (12) lateral fifth MTPs, and (13) lateral calcanei. A single marker was placed on the sacrum. Markers were placed on the prosthesis at similar locations as the sound limb. Participants wore the same shoes for all trials. This ensured consistency of marker placement between trials as all markers were then located either on the socket or the shoe and none on any of the components that were exchanged. The only exception was the lateral ankle, which we attempted to maintain in a similar position by measuring with a caliper relative to the feet markers. Three-dimensional movement of the reflective markers was recorded with an eight-camera motion-capture system at $60 \mathrm{~Hz}$ (Motion Analysis Corp; Santa Rosa, California). Lower-limb joint angle flexion/ extension time series for each joint covering the duration of the entire $3 \mathrm{~min}$ were then calculated from the raw marker position data using custom MATLAB software (MathWorks Inc; Natick, Massachusetts). This time frame was deemed sufficient to properly characterize the walking dynamics based on previous work [18,21-22,26] and is considerably longer than the approximately 7 strides found adequate by Sloot et al. [27].

\section{Dependent Variables}

\section{Largest Lyapunov Exponent}

The stride-to-stride fluctuations in sagittal plane joint angles present during the walking trials were evaluated using the LyE. The LyE was chosen because it is ideal for inherently quasi-periodic signals (i.e., limit cycle) [2122]. Joint kinematic patterns are inherently quasiperiodic. This inherent quasi-periodicity can largely influence other nonlinear measures such as detrended fluctuation analysis and entropy measures and dominate their outcome rather than giving information on the fluctuations that exist on top of these intercycle dynamics. LyE, on the other hand, is ideal for exploring divergence of movement trajectories in state space and especially of those movements that exhibit a limit cycle type of behavior, as in joint kinematics. Thus, the LyE serves well to explore the fluctuations from one stride to the next without the outcome being affected by the inherent quasiperiodicity of joint kinematic patterns. The LyE measures the exponential divergence of the movement trajectories within the reconstructed state space (Figure 2) [14,2830]. State space reconstruction is a nonlinear dynamics technique that uses a time delay $(\tau)$ to create $M$ copies of the original time series, where $M$ is the embedding dimension (Figure 3) [21,28-29]. 


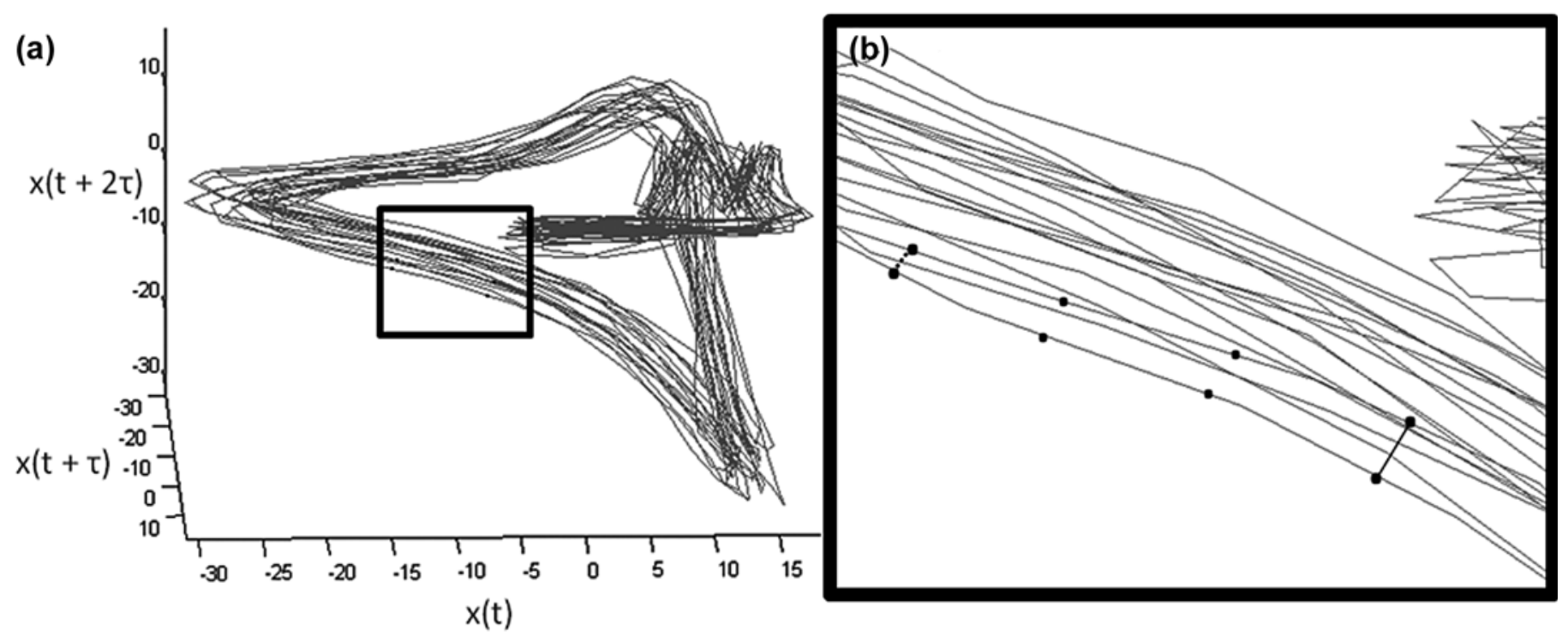

Figure 2.

Largest Lyapunov exponent (LyE) measures divergence of local trajectories in reconstructed attractor. Attractor is region within state space to which all points sufficiently close are drawn toward. (a) Participant's ankle angle time series, as observed in Figure 1, is reconstructed (only three dimensions for visual purpose; actual reconstruction may require more dimensions). In order to quantify divergence, nearest neighbor to point on reference trajectory is selected and allowed to propagate through attractor. Initial distance between points is calculated, and then distance between points is recalculated after propagating through attractor. (b) Zooming in on portion of attractor in which current reference trajectory point is located, it is possible to see selected nearest neighbor and distance between these points (dashed line). After points propagate through attractor for 3 steps, distance between points is calculated again (solid line). Local Lyapunov exponent is then calculated as logarithm base two of ratio of length of solid line to dashed line, normalized to number of propagation steps times amount of time between data points. This process is repeated through entire time series, with LyE being long-time average of these local Lyapunov exponents.

The time delay is calculated through the average mutual information algorithm [31]. The average mutual information calculates the probability that the information within the time-delayed copies of the original time series is different [21,28,31]. Each potential time delay is tested, and then the proper delay is determined to be when the probability reaches the first local minimum. The appropriate embedding dimension is determined through the false nearest neighbors algorithm [14,28,31]. This false nearest neighbors procedure creates multiple time-delayed copies of the original time series, at which point the percentage of "false nearest neighbors" is calculated. A false nearest neighbor is a point that appears close in lower dimensions but is found to be at increased distance when the time series is unfolded to a larger dimension (Figure 4). The proper dimension is the dimension in which the percentage of false nearest neighbors drops to zero [14].
Once the time delay and embedding dimension have been properly determined, the reconstructed state space vector can be obtained (Equation (1)):

$y(t)=[x(t), x(t+\tau), x(t+2 \tau), \ldots x(t+(M-1) \tau)]$,

where $y(t)$ represents the $M$-dimensional coordinates for the reconstructed point at time $t$ derived from the original data point $x$ at time $t(x(t))$. From the reconstructed state space, the LyE can be acquired as a measure of the average divergence of neighboring trajectories. The oscillatory nature of gait results in each stride approximating to an orbit of the attractor. An attractor is the region within the state space to which all points sufficiently close are drawn toward. This appears as a region or "neighborhood" in which all orbits are found (Figure 2) [32]. The LyE is calculated by propagating through the attractor on a single reference trajectory. While moving through the reference 


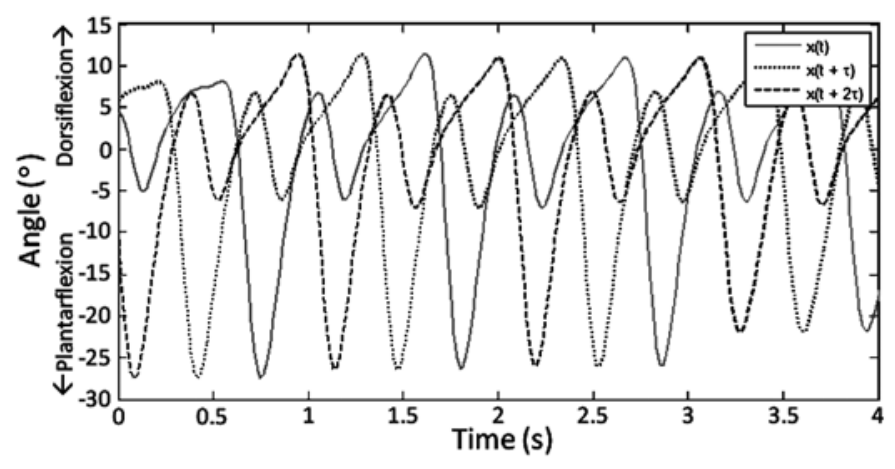

Figure 3.

Ankle angle time series from Figure $\mathbf{1}$ is plotted against time. In addition, copy of time series at single time delay $(\tau)$ as well as copy at two times delay (2T). Number of delay copies is dictated by embedding dimension. This time series has been embedded to three dimensions. Attractor could be plotted as $x(t)$ versus $x(t+T)$ versus $x(t+2 T)$.

trajectory, the nearest neighbor on a different trajectory is selected [29]. The Euclidean distance ( $d t$ ) between the reference point and nearest neighbor is calculated. Then the two points are allowed to propagate and evolve through their specific trajectories a certain amount of time, at which point the evolved distance $\left(d t^{\prime}\right)$ between the points is calculated [29]. The local expansion/contraction rate $(Z)$ at that instant is then calculated as-

$$
Z=\log _{2}\left(d t^{\prime} / d t\right)
$$

$Z$ is then normalized to time by dividing the time between data points $(1 / 60 \mathrm{~s})$ multiplied by the number of points that were propagated through the trajectory [29]. Subsequently, the long-time average of the running sum of the normalized $Z$ values is calculated [29]. At this point, the nearest neighbor is replaced by a new nearest neighbor lying closer to the reference trajectory and the process of propagation and distance calculation is repeated. This continues until the propagation has moved through the entire time series [29]. The long-time average of the $Z$ values once the entire time series has been propagated is the LyE [29]. Input parameters required for calculation of LyE were set at $n=3$ (number of time points to propagate before finding new nearest neighbor $[21,29]$ ), maximum angle of 0.3 radians (maximum angle from reference trajectory in which new nearest neighbor
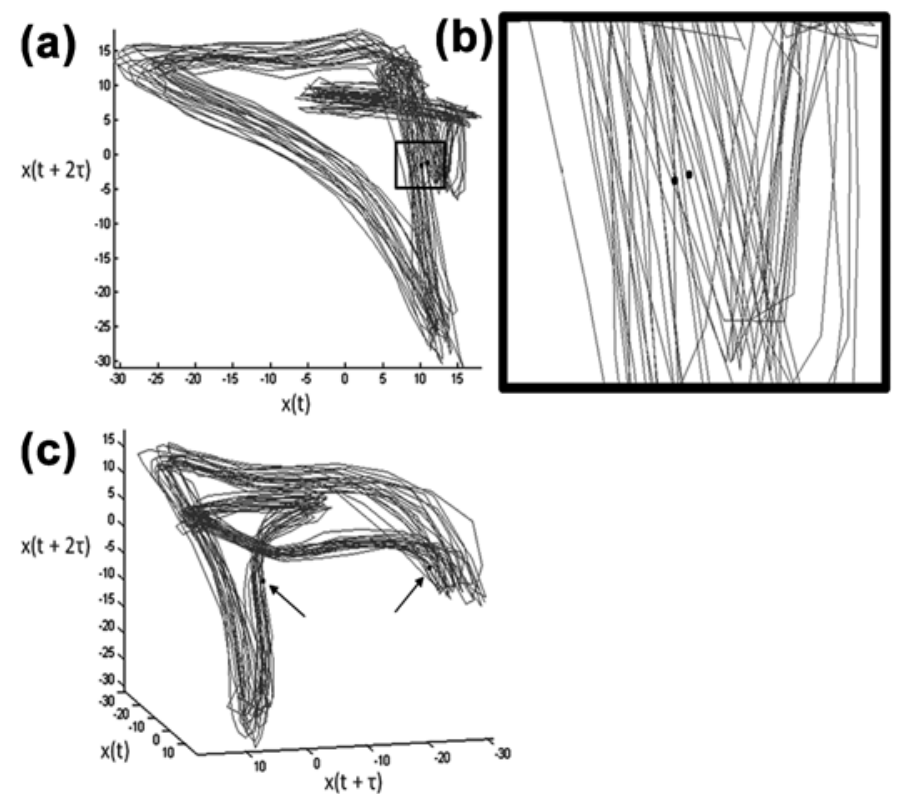

Figure 4.

False nearest neighbor can be seen in this exemplary plot. (a) Ankle angle time series from Figure $\mathbf{1}$ has been embedded into two dimensions. Two-dimensional visual, however, can deceive when determining points that are lying close together. (b) Zooming in on selected points from (a), points appear to be close in their spatial separation. (c) Further including another dimension of attractor, it now becomes obvious that points were in fact false nearest neighbors and are not close. Including more dimensions may reveal more false nearest neighbors; however, eventually false nearest neighbors will no longer exist and only true nearest neighbors will be present. Dimension that drops false nearest neighbors to zero is appropriate embedding dimension.

must reside [29]), minimum scale length of 0.0001 (minimum distance to selection of new nearest neighbor [29]), and maximum scale length of 0.1 times the maximum length of the attractor (maximum distance to selection of new nearest neighbor [29]).

It is important to note that another algorithm is available to calculate the LyE [33]. While the algorithm proposed by Rosenstein et al. [33] has been used to calculate LyE in gait studies [17-18,20,28,30,34], this algorithm differs in that it does not provide a single value. Rather, it calculates local divergence values, plots the natural log of these values against time, and then requires a user selection [33]. The slope of the range of values for the user selection is the estimated LyE [33]. Since the overall 
purpose of this study is to determine a measure that could be implemented in a clinical setting, requiring user selection from a clinician increases the difficulty of introducing such a measure into a clinical setting. As such, the single value output from the Wolf et al. [29] algorithm was deemed a more appropriate method for this study's purpose.

\section{Preference Scale}

The participants' preference between prostheses was assessed through a continuous visual analog scale (Figure 5). Previous research studying preference between prostheses has mainly used a categorical approach $[7,35-36]$. Such an approach leads to decreased resolution, forcing individuals to contain their preference to the specified categories. In particular, this is a major problem if the preference is only between two items (Rx vs Ax prostheses). Furthermore, the LyE is a continuous measure, and as such, it was possible that some individuals would experience a greater change in the LyE between the two prostheses. A greater positive change would indicate more fluctuations with the nonpreferred prosthesis, whereas a greater negative change would indicate more fluctuations with the preferred prosthesis. Larger LyE values are typically associated with a

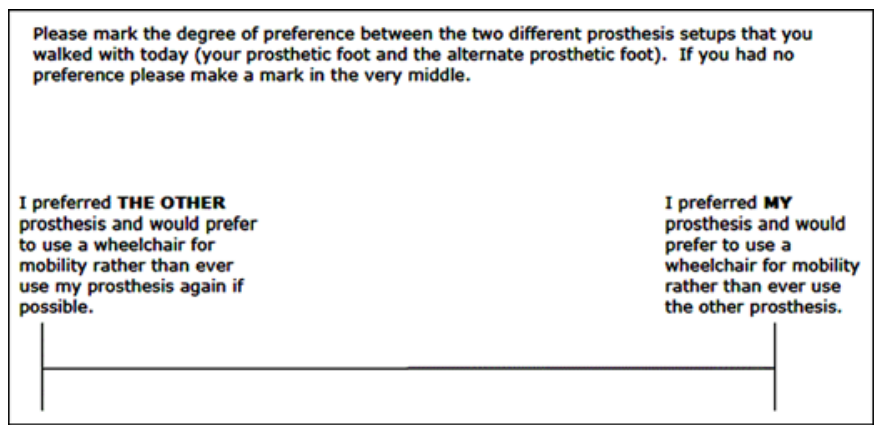

Figure 5.

Visual analog scale designed to assess preference between prostheses. Participants were told to make single mark indicating their preference between two prostheses. Instructions included explanation that if participant had no preference, he or she should make mark in very middle. Attached to extreme ends is inclusion of statement "prefer to use a wheelchair for mobility rather than ..." This was added to prevent floor and ceiling effects, requiring participants to gauge their level of preference rather than simply putting binary response. Responses were then normalized as percentage from left to right for further statistical analysis. process that has increased noise and variance [14]. Based on our hypothesis, a greater change in the LyE should coincide with a greater sense of prosthesis preference. Furthermore, the visual analog preference scale was designed with an added statement of "I preferred MY [THE OTHER] prosthesis and would prefer to use a wheelchair for mobility rather than ever use the other [my] prosthesis . . ." attached to each extreme end in order to avoid floor and ceiling effects. This was based upon anecdotal clinical experience noting that patients with amputation who are currently walking (inclusion criteria) typically desire a prosthesis, even if uncomfortable, before resorting to a wheelchair as their primary form of mobility. Subjects were presented with the visual analog scale after conclusion of the second trial and asked to mark the degree of preference with the noted extremes. Participants were asked prior to scribing their mark on the scale to verbalize the instructions so as to assure comprehension of the task.

Preference was calculated as the percentage distance from the middle point on the scale (point of no preference between prostheses) to the nearest extreme end (point of greatest preference between prostheses). In other words, if the individual marked on the right side of the middle of the preference scale (Figure 5), indicating preference for the $\mathrm{Rx}$ prosthesis, preference was calculated as the percentage distance from the middle of the scale to the extreme end. If, on the other hand, the individual made a mark to the left of the middle point on the analog scale, indicating preference for the Ax prosthesis, preference was calculated as the percentage distance to the extreme end for the Ax prosthesis. This meant a value of 0 percent corresponded to no preference between prostheses, whereas a value of 100 percent would be maximum preference for one of the prostheses. This was done as the study was only concerned with quantifying the amount of preference for a prosthesis (or indifference) and relating this to the change in stride-to-stride fluctuations, rather than comparing preference between prostheses. Values in between corresponded to varying degrees of preference.

\section{Statistical Analysis}

A two-tailed Pearson moment correlation coefficient was used for testing significant correlation between the change in stride-to-stride fluctuations (difference in LyE between Rx and Ax prosthesis) and the degree of preference. Normality of data was assessed using a Shapiro-Wilk test, which confirmed normality of all dependent variables. The preference was then correlated with the value obtained 
through subtraction of the LyE for the preferred prosthesis from the nonpreferred prosthesis. A greater LyE value corresponds with greater divergence between neighboring trajectories within the attractor (Figure 2) [21,29]. We hypothesized that the change in the LyE at the lower-limb joints would be correlated with the prosthesis preference. Significance was set at the 0.05 level.

\section{RESULTS}

Of the 14 individuals recruited, 13 were able to successfully complete the testing. One individual could not obtain a proper fitting with the Ax prosthesis and thus was unable to perform the Ax prosthesis trial. The remaining 13 individuals successfully completed both trials as well as the analog preference scale. All participants had been wearing their current prosthesis for less than a year. The LyE values for all six lower-limb joints were calculated for both conditions (Table 2).

The differences in the LyE and the degree of preference between the prostheses were significantly and positively correlated at the prosthetic ankle $(r=0.629, p=$ 0.02 ; Figure 6). This is a strong or large relationship as defined by Cohen [37]. Nonsignificant correlations reflecting moderate to weak relationships were found for the amputated side knee joint $(r=0.109, p=0.72)$, amputated side hip joint $(r=-0.222, p=0.47)$, sound leg ankle $(r=-0.218, p=0.47)$, sound leg knee joint $(r=-0.141$, $p=0.65)$, and sound leg hip joint $(r=-0.176, p=0.57)$ for the difference in the LyE and the degree of preference between the prostheses (Table 3).

\section{DISCUSSION}

The purpose of this study was to examine the strideto-stride fluctuations in gait for individuals with amputation and determine whether they were related to the indi-

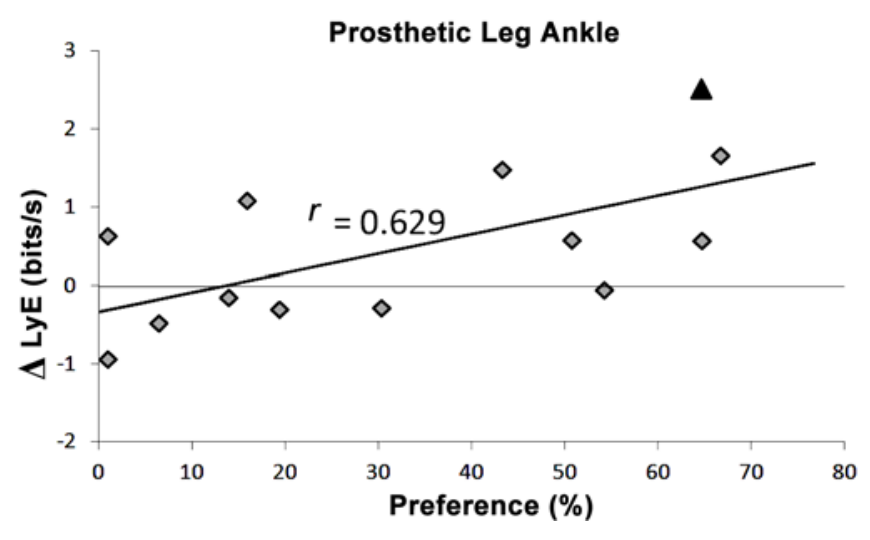

Figure 6.

Difference in largest Lyapunov exponent (LyE) value at prosthetic ankle from preferred prosthesis to nonpreferred prosthesis was significantly correlated with preference between prostheses (difference in LyE calculated as LyE value for walking with nonpreferred prosthesis minus LyE value for walking with preferred prosthesis). This was noted to be strong relationship. This was also true for one participant who ultimately had stronger preference for alternate prosthesis (participant denoted by $\mathbf{\Lambda}$ ).

vidual's perception of the prosthesis. This was done by attempting to invoke a change in stride-to-stride fluctuations and then quantifying the LyE and degree of preference. Our results at the prosthetic ankle support our hypothesis. Stride-to-stride fluctuations at the prosthetic ankle are related to the patient's perception. Specifically, a larger change in the LyE at the prosthetic ankle when walking in a different prosthesis setup was accompanied by greater feelings of preference for one prosthesis over the other. Individuals who had more indifference between the prostheses had less change in the LyE at the prosthetic ankle. This seems to indicate that individuals with amputation have a preferred movement pattern at the prosthetic ankle when walking, one that is more highly organized, leading to decreased stride-to-stride fluctuations. Visual

Table 2.

Group means and standard deviation for largest Lyapunov exponent at each joint with different prosthesis setups: prescribed (Rx) and alternate (Ax). Data presented in bits/second.

\begin{tabular}{lccccccc}
\hline \multirow{2}{*}{ Prosthesis } & \multicolumn{3}{c}{ Amputated Leg } & & \multicolumn{3}{c}{ Sound Leg } \\
\cline { 2 - 4 } & Hip & Knee & Ankle & & Hip & Knee & Ankle \\
\hline Prescribed (Rx) & $1.036 \pm 0.232$ & $1.225 \pm 0.291$ & $2.682 \pm 1.000$ & & $1.200 \pm 0.635$ & $1.322 \pm 0.459$ & $2.238 \pm 0.768$ \\
Alternate (Ax) & $0.923 \pm 0.281$ & $1.211 \pm 0.380$ & $2.885 \pm 1.191$ & & $1.154 \pm 0.517$ & $1.401 \pm 0.381$ & $1.845 \pm 0.510$ \\
\hline \hline
\end{tabular}


Table 3.

Participants' preference between different prosthesis setups, as well as change in largest Lyapunov exponent (LyE) at all six major lower-limb joints. Preference of 100 percent would correspond to maximum preference for one prosthesis over another. Preference of 0 percent would correspond to no preference between prostheses. Preference between prostheses was correlated with change in LyE that occurred at each major lower-limb joint. Ankle on amputated leg (i.e., prosthetic ankle) had marked relationship with preference.

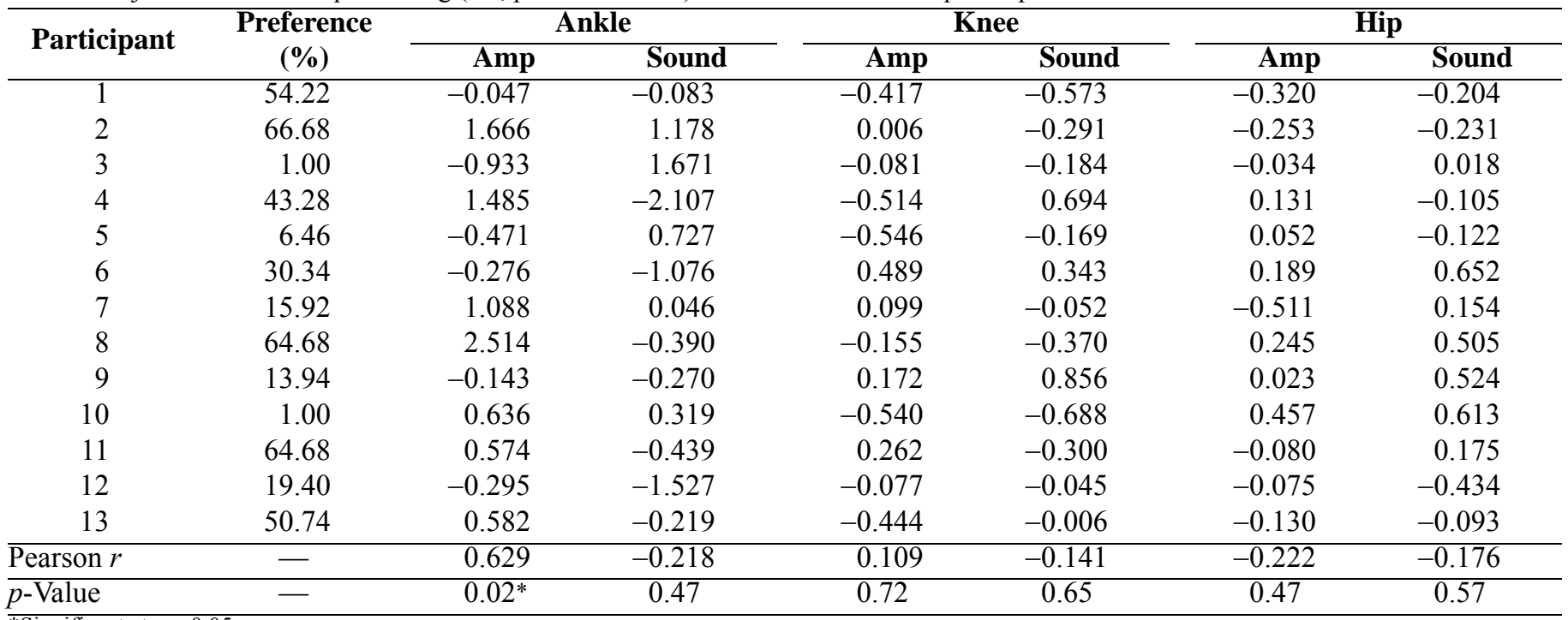

*Significant at $p<0.05$.

Amp $=$ amputated leg, Sound $=$ nonamputated leg.

inspection of the attractor for the prosthetic ankle motion for a "preferred" prosthesis and a "nonpreferred" prosthesis for an example individual display the change in organization of the movement (Figure 7).

In Figure 7(a), the movement pattern that results from better control has a clearly more organized attractor, with trajectories of the attractor falling into a "tighter" path. This is contrasted to Figure 7(b), which displays the "nonpreferred" attractor. In the movement pattern for the nonpreferred attractor, the trajectories are less tightly bunched. This decreased organization reflects greater variance within the state space, which seems to be less desirable in accordance with the preference relationship found here.

The alteration to the preferred movement pattern caused by a different prosthesis causes an increase in the stride-to-stride fluctuations. This change is perceived by the individual with an amputation. The reason that such a relationship was noted at the prosthetic ankle, and not the other lower-limb joints, is likely because this is the single joint that serves to act as the union of the biological (i.e., the individual) and artificial systems (i.e., the prosthesis). For the individuals in this study with transtibial amputations, the prosthetic ankle is the sole joint at which a biological segment and mechanical segment meet (the biological segment is lengthened through a pylon). In other words, the prosthetic ankle has the unique position within the kinematic chain to serve as the merging of the two subsystems (biological and artificial), forming a single dynamical system. Thus, for this group of individuals with transtibial amputations, it is reasonable that the prosthetic ankle had the strongest relationship to changes in the system. Other joints, such as the amputated leg's knee, may be affected by things such as socket trimlines or suspension types, but would not serve this unique role as the "linkage" between the two communicating subsystems within the dynamic system (i.e., the ambulating person with an amputation). That perceived change is related to the individual's preference, possibly providing a link between stride-tostride fluctuations and patient perception.

Initially, it would seem unlikely that the motion about the prosthetic ankle would be strongly related to prosthetic preference given the historical perspective that the performance of the five remaining biological joints are strongly affected and need to compensate [38-43]. However, these studies largely focus on energy generation and absorption. Yet, from simple coordination studies involving finger movement, which requires minimal energy [44-45], it is possible to understand that the underlying goal of neuromuscular control is not entirely 

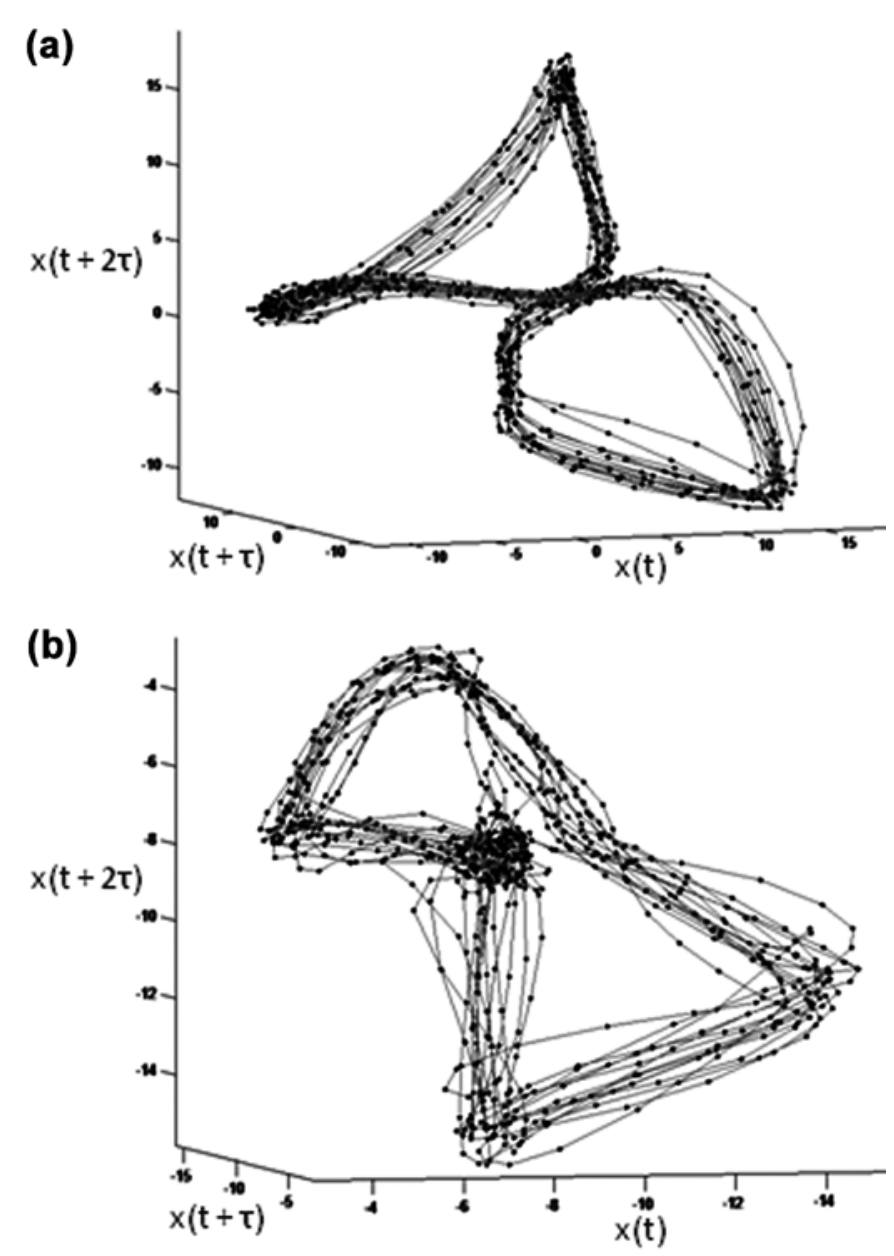

Figure 7.

Prosthesis preference related to change in stride-to-stride fluctuations at prosthetic ankle. Attractors for prosthetic ankle of (a) preferred and (b) nonpreferred prosthesis of an example individual show how an increase in stride-to-stride fluctuations (i.e., increased Lyapunov exponent) reflects greater disorganization. Specifically, note how trajectories of preferred prosthetic ankle movement (a) lie closer together in state space due to more consistent movement. Trajectories of nonpreferred prosthetic ankle movement (b) do not lie as close together, with increased likelihood of stray trajectories that are more dissimilar from previous and future trajectories.

energy management. Hence, when we begin to take a new approach to understanding amputee gait and potential perceptual influences, we may need to consider aspects of movement that have not been traditionally explored (e.g., the subtle fluctuations that occur from stride-to-stride, falling under the larger umbrella term of temporal structure of variability).

It can be seen that a number of individuals (Figure 6), in fact, had greater stride-to-stride fluctuations associated with their preferred prosthesis, resulting in a negative change in LyE between conditions. Furthermore, the correlation coefficient was less than 1 , thereby dictating that other variables are influencing this relationship and account for the remaining variance. It is therefore very plausible that energy management dictated by the prostheses is also influencing the measures (e.g., perhaps while one prosthesis felt like it was producing a smoother motion, the other seemed to have a more efficient energy profile). This unexplained variance could easily shift preference to varying locations on the abscissa. In addition, a major limitation of the setup included a lack of blinding of the participant. It was visible to the participant which prosthesis they had on. Thus, we cannot eliminate the influence of feelings of the "latest and greatest" technology when being able to visibly see a carbon fiber, ESAR type foot and a simple solid-ankle-cushion-heel. Furthermore, because the participants could visibly see whether they were wearing the Rx or Ax prosthesis, we cannot discount potential feelings of ownership associated with the Rx prosthesis. All of this may also likely influence preference. Also, subjective patient report may not be entirely reliable, thus also adding variance to the correlation coefficient. Yet, as mentioned earlier, no studies have previously shown any relationships to prosthesis preference [6]. This is possibly because the attempt to group any of these mentioned factors to get a general trend is difficult because individuals may place greater weight on things like ownership or energy profiles. But, it seems there is a relative weighting with respect to strideto-stride fluctuations that is able to dictate a strong relationship. Thus, we are able to conclude a positive relationship between the stride-to-stride fluctuations of the motion about the prosthetic ankle and prosthesis preference. Further work is still needed to improve this relationship by accounting for the previous factors to hopefully increase the sensitivity of the LyE for proper prescription.

\section{Participant Mr. Doe}

One individual, Participant Mr. Doe, had been ambulating on an ESAR type foot for his current Rx prosthesis. This participant had been using his current $R x$ prosthesis for more than $30 \mathrm{~d}$ without problems. Upon arrival at the laboratory, he expressed satisfaction with 
his current Rx prosthesis. However, after walking trials with both his Rx prosthesis and the Ax prosthesis, he reluctantly informed the investigators that he preferred the Ax prosthesis and marked accordingly on the preference scale (subject noted with $\mathbf{\Delta}$ in Figure 6). The calculation of the LyE showed a change in the LyE that was consistent with the other participants in regards to preference. Whereas other individuals who had a preference for their Rx prostheses had larger LyE values when walking with the Ax prosthesis, Participant Mr. Doe had a decrease in LyE values when walking with the Ax prosthesis. It seems the movement pattern from the Ax prosthesis that is designed for low activity levels allowed stride-to-stride fluctuations that were more preferred by this individual. In other words, while Participant Mr. Doe would seemingly benefit from the many advantages that ESAR feet have been shown to provide $[6,46]$, in his case, the stride-to-stride fluctuations resulting from use of the ESAR foot were not as in sync with his natural movement rhythms. This, however, is speculation as we did not quantify participants' activity levels.

\section{Lyapunov Exponent: Another Tool in Limb-Loss Rehabilitation}

While we feel these findings have provided strong evidence for the study of stride-to-stride fluctuations in individuals with amputation through tools from nonlinear dynamics, specifically the LyE, it is important to note that it should not be viewed as a single metric to define limbloss rehabilitation. The LyE, and possibly other measures of stride-to-stride fluctuations, should be considered important in the rehabilitation of individuals after amputation learning to walk as the changes in the LyE are mirrored by the individuals' sentiment toward their prosthesis. However, the LyE may not detect certain changes within a prosthesis that can be characterized by more typical biomechanical measures. For example, in one of the only two other studies to examine the LyE in individuals with an amputation while walking, Segal et al. reported no significant differences in LyE for individuals walking with and without a torsion adapter [47]. Their participants walked continuously in a circle. It is very possible that a torsion adapter would not affect the stride-to-stride fluctuations. The participants could have fallen in the middle range, with no preference that would correspond to lack of difference in the LyE. But, as stated, the LyE measures the divergence of trajectories within the reconstructed attractor of the walking pattern. It is not concerned with overall magnitude of the movement. It is possible to have similar divergence of trajectories while having different sized trajectories as would be allowed in the movement. In Figure 8(a), two ankle time series from two different individuals display different magnitude differences (Participant A has greater range of motion than Participant B). Figure 8(b) shows the attractors for these time series reconstructed to three dimensions for viewing. The three-dimensional reconstructed attractor for Participant A also spans a larger volume than Participant $\mathrm{B}$; however, the divergence in these attractors is actually very similar at $\mathrm{LyE}$ values of $2.27 \mathrm{bits} / \mathrm{s}$ and $2.24 \mathrm{bits} / \mathrm{s}$. In Segal et al., it is very likely that the torsion adapter altered the magnitude of motion,
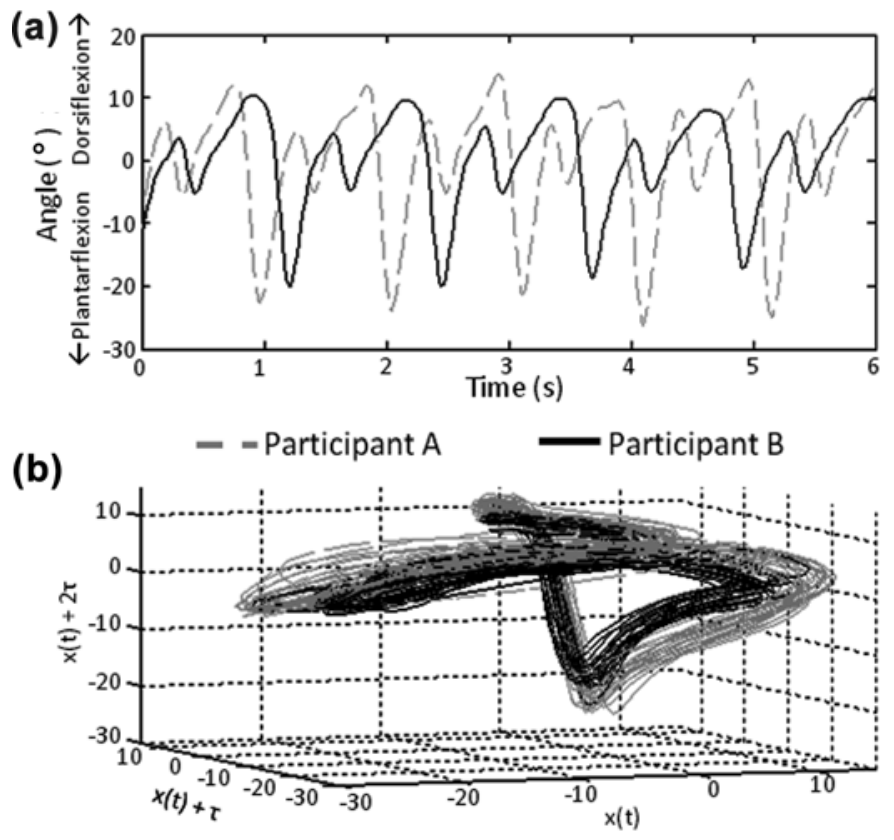

\section{Figure 8.}

Largest Lyapunov exponent calculates variance present within stride-to-stride fluctuations. It does not account for differences in magnitude of movement. (a) Two different ankle angle time series for two different participants. Participant $A$ is walking with increased range of motion at ankle (greater absolute local maxima and minima) compared with Participant B. (b) Inspection of attractors for both time series embedded into three dimensions further supports fact that Participant $A$ is walking with greater range of motion at ankle (seen through larger relative hyperradius of attractor). Yet, these particular time series for these two different ankles have similar divergence of neighboring trajectories within attractor (Lyapunov exponent 2.27 bits/s and $2.24 \mathrm{bits} / \mathrm{s})$. 
but did not necessarily affect the movement pattern within the stride-to-stride fluctuations [47]. The fact that the LyE is not sensitive to movement magnitude may further increase its importance as a prescriptive or outcome measure as it is not sensitive to the magnitude differences expected between different sized individuals, allowing for comparison across heterogeneous populations of individuals with an amputation. Yet, it is also important that traditional biomechanical measures that account for such magnitude differences still be examined.

Lamoth et al. [48] are the only other authors besides Segal et al. [47] to examine LyE in a limb-loss population. Their study examined the LyE in a group of eight individuals with a unilateral, transfemoral amputation. They calculated LyE from an accelerometer attached to the trunk during walking under four different conditions. The comparison of LyE from an accelerometer attached to the trunk and joint angle flexion/extension is not possible. However, it is interesting that Lamoth et al. reported increased LyE values for the individuals with limb loss compared with the nonaffected control subjects [48]. This is consistent with a preference expressed by the current group of individuals in this study for the prosthesis that had decreased LyE values, or values possibly closer to that found in nonaffected control subjects.

\section{Study Limitations}

This study has limitations that should be considered. First, participants were only given a few minutes to walk with the Ax prosthesis before data collection, which may not have been an adequate acclimation period. Such acclimation may have resulted in different LyE values for the Ax prosthesis. In order to compare LyE for the two specific feet, a longer acclimation period would be beneficial. However, the purpose of our study was not to compare the LyE values for two specific feet, but rather to relate the LyE to prosthesis preference. But, if it were to be presumed that acclimation would affect LyE values, then it would likely result in LyE values for the Ax prosthesis approaching values closer to those measured for the participants' Rx prostheses, followed by a plateau. In addition, those prostheses initially at LyE values that are much further away from the value at which they would plateau would require more time/practice (i.e., more rehabilitation) before the person is fully adjusted to the prosthesis. Therefore, starting with a prosthesis that leads to a LyE value closer to the final optimal value could potentially constitute a prescriptive tool. Second, in order to increase subject recruitment, the inclusion criteria did not specify cause of amputation, which has been shown to affect biomechanical measures [8,49-50]. However, this limitation may also be viewed as a strength. Consider that failure to account for such a difference can mask results in studies using more traditional biomechanical measures that average multiple steps to get a single representative step; yet our results showed a strong relationship in a heterogeneous population of individuals with amputations. This seems to indicate the robustness of the LyE in gait for individuals with amputation, a requirement for any potential prescriptive tool or outcome measure. Furthermore, we have found significant findings at the prosthetic ankle, which for the majority of prosthetic feet does not represent a true rotation but more of a "bending." Yet, it is this bending that serves to recreate ankle plantar/dorsiflexion, and it is the consistency of this movement that appears related to prosthesis preference. This significant finding in the bending motion also has the additional benefit of not being as heavily influenced by ankle joint location as would be the case for traditional kinetics and kinematics, especially as seen in inverse dynamics [51]. Similarly, this lack of emphasis on joint location may also help to overcome natural limitations associated with motion capture for limb-loss gait [52]. In addition, we have correlated a change in the LyE with prosthesis preference; however, the minimal clinically important difference for the LyE is not known [53]. Future work should determine this difference pertaining to more traditional functional measures such as the $6 \mathrm{~min}$ walk test and the timed "up-and-go" test.

\section{CONCLUSIONS}

In conclusion, individuals' perceptions of their prostheses seem to be related to the stride-to-stride fluctuations at the prosthetic ankle during gait. This was also the case for an individual who ultimately preferred an Ax prosthesis over his own Rx prosthesis that he had been ambulating with for more than $30 \mathrm{~d}$ without any problems. This study is the first to find a biomechanical measure related to prosthesis preference. A large body of work is needed to further determine the proper implementation in the clinical setting. For example, is it only possible to use the LyE when comparing prostheses or can we compare to a threshold? Thus, future work is needed to better understand the benefits associated with decreased fluctuations from stride-to-stride at the prosthetic ankle for individuals walking with a prosthesis, 
possibly discovering a threshold for positive outcomes. Future work should also be aimed at addressing the effects of acclimation to a prosthesis on the LyE as well as examining which joint is influencing the patient's preference for those individuals with amputation levels other than transtibial and beyond unilateral.

Clinically, we believe that the examination of strideto-stride fluctuations using nonlinear methodology can eventually become a routine practice among prosthetists, therapists, and physiatrists to examine the functional outcome of prosthetic rehabilitation. This, in essence, would be for these clinicians what measuring heart rate variability using Holter devices is for cardiologists. We similarly foresee this to be an easy procedure, which will be based on the use of a handheld and wearable device that can acquire data while the patient is walking. The data from the wearable device (e.g., adhesive electrogoniometer) will be downloaded to the handheld via wireless technology for evaluation. The software loaded on the handheld will be able to analyze the data with nonlinear methodology (i.e., LyE). In this fashion, the rehabilitation team will be able to assess the patient's stride-to-stride fluctuations due to his or her current prosthesis in terms of previous prostheses or a normative database. Furthermore, current trial periods associated with new prosthetic feet lend themselves to quickly making "in-office" assessments among multiple prosthetic feet. Based on these evaluations, combined with more traditional assessments, recommendations for rehabilitation or prosthesis changes can be provided.

\section{ACKNOWLEDGMENTS}

\section{Author Contributions:}

Study concept and design: S. R. Wurdeman, N. Stergiou. Acquisition of data: S. R. Wurdeman.

Analysis and interpretation of data: S. R. Wurdeman, S. A. Myers, A. L. Jacobsen, N. Stergiou.

Drafting of manuscript: S. R. Wurdeman, A. L. Jacobsen. Critical revision of manuscript for important intellectual content: S. R. Wurdeman, S. A. Myers, A. L. Jacobsen, N. Stergiou. Statistical analysis: S. R.Wurdeman, N. Stergiou.

Study supervision: N. Stergiou.

Financial Disclosures: The authors have declared that no competing interests exist. We kindly acknowledge The Ohio Willow Wood Company (Mt. Sterling, Ohio) for generously donating the solid-ankle cushion-heel feet and pylons. The Ohio Willow Wood Company did not have any involvement in the study design; data collection, analysis, or interpretation; and writing or submission of this article.

Funding/Support: This material was based on work supported through a Widaman Fellowship (SRW).
Additional Contributions: We kindly acknowledge Jeffrey Kaipust, MS, for assistance with data acquisition.

Institutional Review Board: All participants provided written informed consent as approved by the University of Nebraska Medical Center Institutional Review Board.

Participant Follow-Up: Those subjects who requested to be informed of the publication of this study will be informed.

\section{REFERENCES}

1. Ziegler-Graham K, MacKenzie EJ, Ephraim PL, Travison TG, Brookmeyer R. Estimating the prevalence of limb loss in the United States: 2005 to 2050. Arch Phys Med Rehabil. 2008;89(3):422-29. [PMID:18295618] http://dx.doi.org/10.1016/j.apmr.2007.11.005

2. Dillingham TR, Pezzin LE, MacKenzie EJ. Limb amputation and limb deficiency: epidemiology and recent trends in the United States. South Med J. 2002;95(8):875-83. [PMID:12190225]

3. Gailey RS, Roach KE, Applegate EB, Cho B, Cunniffe B, Licht S, Maguire M, Nash MS. The amputee mobility predictor: an instrument to assess determinants of the lowerlimb amputee's ability to ambulate. Arch Phys Med Rehabil. 2002;83(5):613-27. [PMID:11994800]

4. van der Linde H, Hofstad CJ, Geurts AC, Postema K, Geertzen JH, van Limbeek J. A systematic literature review of the effect of different prosthetic components on human functioning with a lower-limb prosthesis. J Rehabil Res Dev. 2004;41(4):555-70. [PMID:15558384] http://dx.doi.org/10.1682/JRRD.2003.06.0102

5. Smith DG, Michael JW, Bowker JH. Atlas of amputations and limb deficiencies: Surgical, prosthetic, and rehabilitation principles. 3rd ed. Rosemont (IL): American Academy of Orthopedic Surgeons; 2004.

6. Hafner BJ, Sanders JE, Czerniecki J, Fergason J. Energy storage and return prostheses: does patient perception correlate with biomechanical analysis? Clin Biomech (Bristol, Avon). 2002;17(5):325-44. [PMID:12084537] http://dx.doi.org/10.1016/S0268-0033(02)00020-7

7. Macfarlane PA, Nielsen DH, Shurr DG, Meier K. Perception of walking difficulty by below-knee amputees using a conventional foot versus the Flex-Foot. J Prosthet Orthot. 1991;3:114-19.

8. Torburn L, Powers CM, Guiterrez R, Perry J. Energy expenditure during ambulation in dysvascular and traumatic below-knee amputees: a comparison of five prosthetic feet. J Rehabil Res Dev. 1995;32(2):111-19. [PMID:7562650]

9. Kark L, Simmons A. Patient satisfaction following lowerlimb amputation: the role of gait deviation. Prosthet Orthot Int. 2011;35(2):225-33. [PMID:21558305] http://dx.doi.org/10.1177/0309364611406169 
10. Hausdorff JM. Gait dynamics, fractals and falls: finding meaning in the stride-to-stride fluctuations of human walking. Hum Mov Sci. 2007;26(4):555-89. [PMID:17618701] http://dx.doi.org/10.1016/j.humov.2007.05.003

11. Stergiou N, Decker LM. Human movement variability, nonlinear dynamics, and pathology: is there a connection? Hum Mov Sci. 2011;30(5):869-88. [PMID:21802756] http://dx.doi.org/10.1016/j.humov.2011.06.002

12. Decker LM, Cignetti F, Stergiou N. [Complexity and human gait]. Rev Andal Med Deporte. 2010;3:2-12. Spanish.

13. Forner Cordero A, Koopman HF, van der Helm FC. Multiple-step strategies to recover from stumbling perturbations. Gait Posture. 2003;18(1):47-59. [PMID:12855300] http://dx.doi.org/10.1016/S0966-6362(02)00160-1

14. Stergiou N, Buzzi UH, Kurz MJ, Heidel J. Nonlinear tools in human movement. In: Stergiou N, editor. Innovative analyses of human movement. Champaign (IL): Human Kinetics; 2003. p. 63-90.

15. Stergiou N, Harbourne R, Cavanaugh J. Optimal movement variability: a new theoretical perspective for neurologic physical therapy. J Neurol Phys Ther. 2006;30(3): 120-29. [PMID:17029655]

16. Goldstein EB. Sensation \& perception. 8th ed. Canada: Wadsworth Pub Co/Cengage Learning; 2009.

17. Bruijn SM, Bregman DJ, Meijer OG, Beek PJ, van Dieën $\mathrm{JH}$. Maximum Lyapunov exponents as predictors of global gait stability: a modelling approach. Med Eng Phys. 2012; 34(4):428-36. [PMID:21856204] http://dx.doi.org/10.1016/j.medengphy.2011.07.024

18. Bruijn SM, Meijer OG, Beek PJ, van Dieën JH. The effects of arm swing on human gait stability. J Exp Biol. 2010; 213(Pt 23):3945-52. [PMID:21075935] http://dx.doi.org/10.1242/jeb.045112

19. Buzzi UH, Stergiou N, Kurz MJ, Hageman PA, Heidel J. Nonlinear dynamics indicates aging affects variability during gait. Clin Biomech (Bristol, Avon). 2003;18(5):435-43. [PMID:12763440] http://dx.doi.org/10.1016/S0268-0033(03)00029-9

20. Nessler JA, Huynh H, McDougal M. A single bout of resistance exercise does not affect nonlinear dynamics of lower extremity kinematics during treadmill walking. Gait Posture. 2011;34(2):285-87. [PMID:21570292] http://dx.doi.org/10.1016/j.gaitpost.2011.04.003

21. Myers SA, Johanning JM, Stergiou N, Celis RI, Robinson L, Pipinos II. Gait variability is altered in patients with peripheral arterial disease. J Vasc Surg. 2009;49(4):924-31, e1. [PMID:19217749] http://dx.doi.org/10.1016/j.jvs.2008.11.020

22. Myers SA, Pipinos II, Johanning JM, Stergiou N. Gait variability of patients with intermittent claudication is similar before and after the onset of claudication pain. Clin Biomech (Bristol, Avon). 2011;26(7):729-34.

\section{[PMID:21450380]}

http://dx.doi.org/10.1016/j.clinbiomech.2011.03.005

23. Jordan K, Newell KM. The structure of variability in human walking and running is speed-dependent. Exerc Sport Sci Rev. 2008;36(4):200-204. [PMID:18815489]

http://dx.doi.org/10.1097/JES.0b013e3181877d71

24. Edwards M. Northwestern University Prosthetics Program: Transtibial manual. Chicago (IL): Northwestern University; 1996.

25. Vaughan CL, Davis BL, O'Connor JC. Dynamics of human gait. Champaign (IL): Human Kinetics; 1992.

26. Bruijn SM, van Dieën JH, Meijer OG, Beek PJ. Is slow walking more stable? J Biomech. 2009;42(10):1506-12. [PMID:19446294] http://dx.doi.org/10.1016/j.jbiomech.2009.03.047

27. Sloot LH, van Schooten KS, Bruijn SM, Kingma H, Pijnappels M, van Dieën JH. Sensitivity of local dynamic stability of over-ground walking to balance impairment due to galvanic vestibular stimulation. Ann Biomed Eng. 2011; 39(5):1563-69. [PMID:21222163] http://dx.doi.org/10.1007/s10439-010-0240-y

28. Kurz MJ, Judkins TN, Arellano C, Scott-Pandorf M. A passive dynamic walking robot that has a deterministic nonlinear gait. J Biomech. 2008;41(6):1310-16.

[PMID:18359030]

http://dx.doi.org/10.1016/j.jbiomech.2008.01.007

29. Wolf A, Swift JB, Swinney HL, Vastano JA. Determining Lyapunov exponents from a time series. Physica D. 1985; $16: 285-317$. http://dx.doi.org/10.1016/0167-2789(85)90011-9

30. Nessler JA, De Leone CJ, Gilliland S. Nonlinear time series analysis of knee and ankle kinematics during side by side treadmill walking. Chaos. 2009;19(2):026104.

[PMID:19566264] http://dx.doi.org/10.1063/1.3125762

31. Abarbanel HD. Analysis of observed chaotic data. New York: Springer-Verlag; 1996.

32. Milnor J. On the concept of attractor. Commun Math Phys. 1985;99:177-95. http://dx.doi.org/10.1007/BF01212280

33. Rosenstein MT, Collins JJ, DeLuca CJ. A practical method for calculating largest Lyapunov exponents from small data sets. Physica D. 1993;65:117-34.

http://dx.doi.org/10.1016/0167-2789(93)90009-P

34. Terrier P, Dériaz O. Kinematic variability, fractal dynamics and local dynamic stability of treadmill walking. J Neuroeng Rehabil. 2011;8:12. [PMID:21345241]

http://dx.doi.org/10.1186/1743-0003-8-12

35. Alaranta H, Kinnunen A, Karkkainen M, Pohjolainen T, Heliovaara M. Practical benefits of Flex-Foot in belowknee amputees. J Prosthet Orthot. 1991;3:179-81. http://dx.doi.org/10.1097/00008526-199100340-00011 
36. Klodd E, Hansen A, Fatone S, Edwards M. Effects of prosthetic foot forefoot flexibility on gait of unilateral transtibial prosthesis users. J Rehabil Res Dev. 2010;47(9):899-910. [PMID:21174254] http://dx.doi.org/10.1682/JRRD.2009.10.0166

37. Cohen J. Statistical power analysis for the behavioral sciences. 2nd ed. Hillsdale (NJ): Lawrence Erlbaum Associates; 1988.

38. Grumillier C, Martinet N, Paysant J, André JM, Beyaert C. Compensatory mechanism involving the hip joint of the intact limb during gait in unilateral trans-tibial amputees. J Biomech. 2008;41(14):2926-31. [PMID:18771768] http://dx.doi.org/10.1016/j.jbiomech.2008.07.018

39. Powers CM, Torburn L, Perry J, Ayyappa E. Influence of prosthetic foot design on sound limb loading in adults with unilateral below-knee amputations. Arch Phys Med Rehabil. 1994;75(7):825-29. [PMID:8024435]

40. Prinsen EC, Nederhand MJ, Rietman JS. Adaptation strategies of the lower extremities of patients with a transtibial or transfemoral amputation during level walking: a systematic review. Arch Phys Med Rehabil. 2011;92(8):1311-25.

[PMID:21714957] http://dx.doi.org/10.1016/j.apmr.2011.01.017

41. Sagawa Y Jr, Turcot K, Armand S, Thevenon A, Vuillerme $\mathrm{N}$, Watelain E. Biomechanics and physiological parameters during gait in lower-limb amputees: a systematic review. Gait Posture. 2011;33(4):511-26. [PMID:21392998] http://dx.doi.org/10.1016/j.gaitpost.2011.02.003

42. Silverman AK, Fey NP, Portillo A, Walden JG, Bosker G, Neptune RR. Compensatory mechanisms in below-knee amputee gait in response to increasing steady-state walking speeds. Gait Posture. 2008;28(4):602-9.

[PMID:18514526] http://dx.doi.org/10.1016/j.gaitpost.2008.04.005

43. Vanicek N, Strike SC, McNaughton L, Polman R. Lower limb kinematic and kinetic differences between transtibial amputee fallers and non-fallers. Prosthet Orthot Int. 2010; 34(4):399-410. [PMID:20450461] http://dx.doi.org/10.3109/03093646.2010.480964

44. Scholz JP, Kelso JA. Intentional switching between patterns of bimanual coordination depends on the intrinsic dynamics of the patterns. J Mot Behav. 1990;22(1):98-124. [PMID:15111283]

45. Scholz JP, Kelso JA. A quantitative approach to understanding the formation and change of coordinated movement patterns. J Mot Behav. 1989;21(2):122-44. [PMID:15132941]

46. Hafner BJ, Sanders JE, Czerniecki JM, Fergason J. Transtibial energy-storage-and-return prosthetic devices: a review of energy concepts and a proposed nomenclature. J Rehabil Res Dev. 2002;39(1):1-11. [PMID:11926321]
47. Segal AD, Orendurff MS, Czerniecki JM, Shofer JB, Klute GK. Local dynamic stability of amputees wearing a torsion adapter compared to a rigid adapter during straight-line and turning gait. J Biomech. 2010;43(14):2798-2803.

[PMID:20719315]

http://dx.doi.org/10.1016/j.jbiomech.2010.05.038

48. Lamoth CJ, Ainsworth E, Polomski W, Houdijk H. Variability and stability analysis of walking of transfemoral amputees. Med Eng Phys. 2010;32(9):1009-14.

[PMID:20685147]

http://dx.doi.org/10.1016/j.medengphy.2010.07.001

49. Casillas JM, Dulieu V, Cohen M, Marcer I, Didier JP. Bioenergetic comparison of a new energy-storing foot and SACH foot in traumatic below-knee vascular amputations. Arch Phys Med Rehabil. 1995;76(1):39-44.

[PMID:7811172]

http://dx.doi.org/10.1016/S0003-9993(95)80040-9

50. Snyder RD, Powers CM, Fontaine C, Perry J. The effect of five prosthetic feet on the gait and loading of the sound limb in dysvascular below-knee amputees. J Rehabil Res Dev. 1995;32(4):309-15. [PMID:8770795]

51. Sawers A, Hahn ME. Trajectory of the center of rotation in non-articulated energy storage and return prosthetic feet. J Biomech. 2011;44(9):1673-77. [PMID:21481878] http://dx.doi.org/10.1016/j.jbiomech.2011.03.028

52. Rusaw D, Ramstrand N. Motion-analysis studies of transtibial prosthesis users: a systematic review. Prosthet Orthot Int. 2011;35(1):8-19. [PMID:21515885] http://dx.doi.org/10.1177/0309364610393060

53. Jaeschke R, Singer J, Guyatt GH. Measurement of health status. Ascertaining the minimal clinically important difference. Control Clin Trials. 1989;10(4):407-15. [PMID:2691207] http://dx.doi.org/10.1016/0197-2456(89)90005-6

Submitted for publication June 1, 2012. Accepted in revised form October 29, 2012.

This article and any supplemental material should be cited as follows:

Wurdeman SR, Myers SA, Jacobsen AL, Stergiou N. Prosthesis preference is related to stride-to-stride fluctuations at the prosthetic ankle. J Rehabil Res Dev. 2013; 50(5):671-86.

http://dx.doi.org/10.1682/JRRD.2012.06.0104

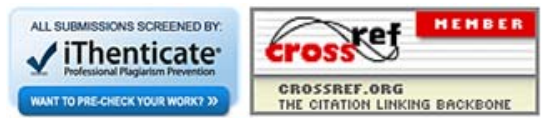


